\title{
OZONIZACIÓN DE AGUAS REGENERADAS EN POMELO 'STAR RUBY'. COMPORTAMIENTO DE LA INSTALACIÓN Y RESPUESTA AGRONÓMICA Y FISIOLÓGICA DEL CULTIVO
}

\author{
Botía Ordaz, P. (1) (P), Hernandez Ballester, FM. (1), Robles García, JM (1), Olivares \\ Quilez, L. (1), Palazón López, J.A.(1), Arques Pardo, E.M. (1), Martínez Izquierdo, A.V. (1), \\ Romero Azorín, P.(1), Berríos,P.(2), Gil Fernández, I.(2), Pérez-Pastor,A.(2), Navarro
}

Acosta, JM. (1)

\author{
Equipo de Riego y Fisiología del Estrés (IMIDA), C/Mayor s/n, La Alberca (Murcia). E-mail: \\ pablo.botia@carm.es \\ Departamento de Ingeniería Agronómica, Universidad Politécnica de Cartagena, Cartagena \\ (Murcia) E-mail: alex.perez-pastor@edu.upct.es
}

2

\section{Resumen}

Durante la campaña 2019-2020, se llevó a cabo un experimento para evaluar el comportamiento de una instalación de ozonización a pie de parcela cuando se utilizan aguas de diferente naturaleza (Regenerada y Trasvase) y obtener resultados preliminares a nivel agronómico y fisiológico de un cultivo de pomelo en estas condiciones. El ensayo se llevó a cabo, en una finca experimental en Campotejar (Murcia), sobre la variedad 'Star Ruby', empleando un diseño bifactorial (Tipo de agua y Ozonización), con bloques completos al azar y 6 repeticiones por combinación. La aplicación de ozono, se realizó entre los meses de junio y agosto mediante una instalación creada exprofeso, y situada a pie de parcela, utilizando un generador de $\mathrm{O}_{3}$ de $20 \mathrm{gr} / \mathrm{hora}$, procurando mantener una dosis constante del mismo (entre 600 y $650 \mathrm{mV}$ en valor redox a pH 6 a la salida de la instalación). En todos los riegos, durante este periodo, se aplicó el $\mathrm{O}_{3}$ durante todo el tiempo de mismo, menos en la fase de abonado y post-abonado, para evitar posibles efectos indeseables sobre la fertilización. Durante el resto del año, los árboles fueron fertirrigados según criterio del agricultor, utilizando el agua disponible (nunca regenerada). Durante el periodo de estudio, se evaluaron en continuo los valores redox durante todos los eventos de riego, a la entrada y la salida de la instalación, el volumen de agua aplicado, así como algunos parámetros de crecimiento vegetativo y del fruto y estado hídrico del cultivo. Los parámetros de producción y calidad, así como el estado nutricional del cultivo de la campaña, también fueron evaluados. Nuestros resultados denotan que las instalaciones utilizadas para la aplicación de un tratamiento de ozonización a pie de parcela, cuando se utilizan aguas regeneradas, pueden presentar dificultades derivadas de la naturaleza de estas aguas (elevados niveles de materia orgánica y otras sustancias altamente oxidables), lo que hace necesario un estudio más exhaustivo de estos sistemas para poder llevar a cabo un tratamiento eficaz de $\mathrm{O}_{3}$ en estas condiciones. Dimensionar adecuadamente la generación de $\mathrm{O}_{3}$ para cada instalación en función de la naturaleza de las aguas utilizadas e implementar sistemas que garanticen la presurización de la instalación y procuren la dosificación adecuada de medio ácido, pueden ser algunas de las recomendaciones a seguir para este tipo de instalaciones. Por otra parte, la respuesta agronómica y fisiológica, ante estos tratamientos requiere igualmente de un mayor y más largo estudio, para poder alcanzar resultados confiables, dado que en el presente estudio solo se evaluaron los efectos de la aplicación de estos tratamientos durante una etapa muy concreta del desarrollo del fruto. 


\section{1) Introducción}

La falta de disponibilidad de agua en regiones áridas y semiáridas, como la Región de Murcia, hace necesaria la utilización de todos los recursos hídricos disponibles para satisfacer las necesidades de los cultivos. En la Región de Murcia las aguas regeneradas provenientes de las depuradoras apenas suponen $100 \mathrm{Hm}^{3}$ y se utilizan prácticamente en su totalidad en el ámbito agrícola. Sin embargo, aunque esta cantidad, apenas suponen una mínima parte de la cantidad necesaria para completar las necesidades hídricas de los cultivos en esta región, se posiciona como un recurso importante y nada desdeñable, máxime en el momento actual, en el que las fuentes convencionales tradicionales están seriamente mermadas en su cuantía Por lo que esta práctica debe ser incluida dentro de la gestión integral del agua y asumida dentro de la estructura de las instituciones del sector hídrico (Segui, 2004).

Sin embargo, la utilización de aguas regeneradas en agricultura plantea ciertos problemas derivados de la naturaleza de las mismas como elevada salinidad, presencia de niveles de microorganismos y compuestos potencialmente contaminantes, perjudiciales o tóxicos para algunos cultivos (Poysa et al., 1987, Resh, 1997, Assouline y Narkis, 2013) que hacen que el agricultor, muestre ciertas reticencias a la hora de su utilización. Por si fuera poco, la reciente normativa en este ámbito (RD 1620/2007), puede restringir su uso actual, si no se toman medidas adicionales a las adoptadas hasta ahora.

El ozono $\left(\mathrm{O}_{3}\right)$, es uno de los compuestos más oxidantes que existe en la naturaleza. Su poder eficaz de desinfección ha sido demostrado profusamente en numerosos ámbitos, incluido el agrícola, donde ha sido aplicado sobre todo en el sector hortícola, con resultados satisfactorios para producir una desinfección de los cultivos y aumentar el rendimiento de los mismos (Martínez et al., 2013; Segura et al., 2003). Incluso algunos estudios apuntan su idoneidad y efectividad sobre los llamados contaminantes emergentes (Kim et al., 2009; Gerrity y Snyder 2011). Su carácter efímero, hace que deba producirse in situ, para poder utilizarlo en el momento que se precise. En su creación, se deriva además, la producción de oxígeno, el cual podría ser utilizado como una ventaja adicional para los cultivos (Abuarab et al., 2013; Bhattarai et al., 2004; Bhattarai, 2005; Letey, 1961).

\section{2) Objetivos}

El principal objetivo de este estudio es evaluar el comportamiento de una instalación de ozonización a pie de parcela cuando se utilizan aguas de diferente naturaleza, de buena calidad, procedente del Trasvase Tajo-Segura (Trasvase) y procedente de una estación depuradora (Regenerada), así como de obtener resultados preliminares a nivel agronómico y fisiológico del cultivo de pomelo de la variedad 'Star Ruby', regado con aguas de esta naturaleza que han sido ozonizadas.

\section{3) Materiales y Métodos}

El experimento se lleva a cabo en una parcela comercial de pomelos adultos de la variedad 'Star Ruby' sobre el portainjerto Citrus macrophylla Wester, situada en la zona de Campotejar (Murcia), muy cerca de la EDAR de Molina de Segura (Figura 1). 


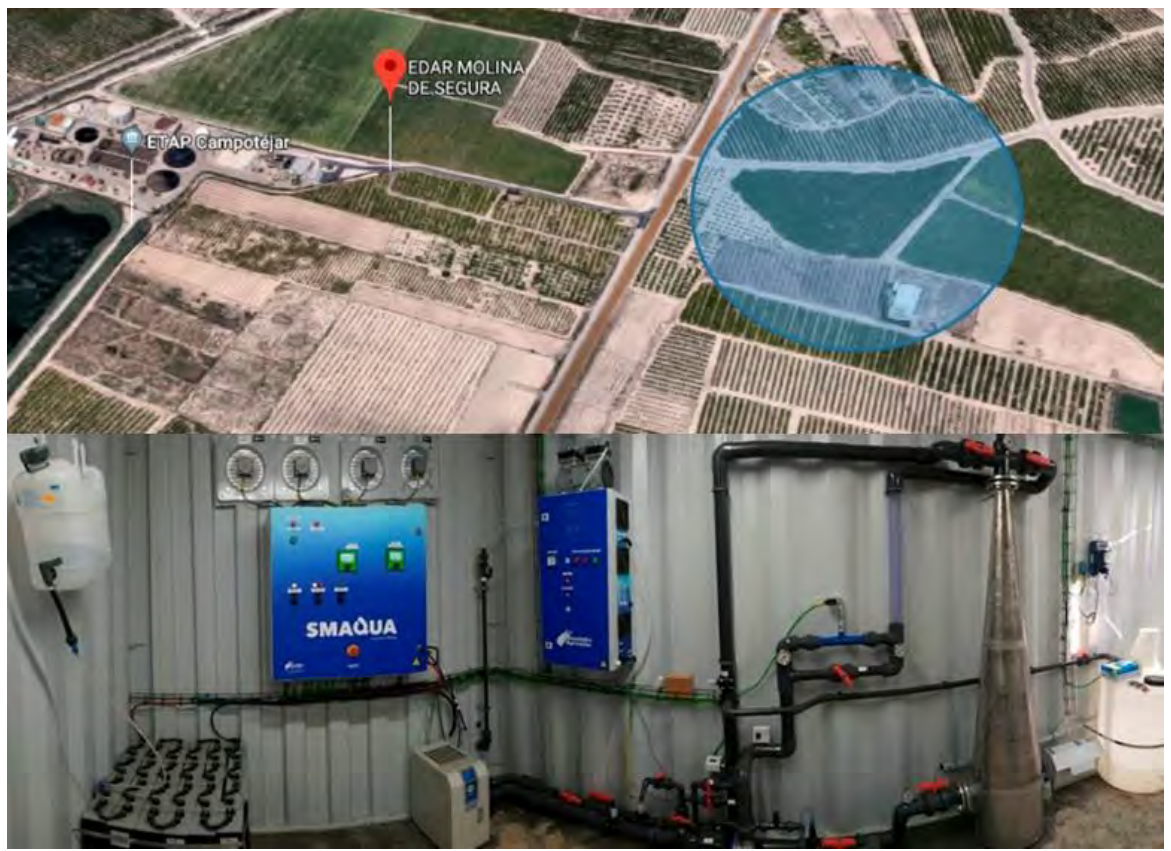

Figura 1. Instalación de ozonización a pie de parcela. Localización y detalle de la instalación.

El estudio se realizó durante el periodo de máxima demanda evaporativa, (junioagosto), coincidente con la fase II de crecimiento del fruto, en la que se aplicó un riego diferencial utilizando aguas de distinta naturaleza (Tabla 1), procedentes del Trasvase TajoSegura (tratamiento $T, C E=1,16 \mathrm{dS} / \mathrm{m}$ ) y aguas regeneradas (tratamiento $R, C E=3,16 \mathrm{dS} / \mathrm{m}$ ) procedentes de la EDAR de Molina de Segura próxima a la parcela experimental, aplicando a su vez para ambos tipos de aguas, un tratamiento de $\mathrm{O}_{3}$, ( $\mathrm{TO}_{3}$ y $\mathrm{RO}_{3}$ respectivamente). Se realizó un diseño bifactorial de bloques completos al azar (con los factores principales Tipo de agua y Ozonización). La programación del riego durante este periodo se realizó en base a la demanda evaporativa, controlando la cantidad de agua finalmente aplicada mediante contadores volumétricos. El plan de riego y abonado durante el resto de la campaña fue procurado por el agricultor según las prácticas habituales de la zona, utilizando el agua en cada momento disponible (normalmente de Trasvase, o mezcla con subterránea, pero nunca de origen regenerada).

La aplicación de ozono se realizó mediante una instalación creada exprofeso, y situada a pie de parcela, utilizando un generador de $\mathrm{O}_{3}$ modelo SMAQUA OZ20 de hasta $20 \mathrm{gr} / \mathrm{hora}$ de generación de $\mathrm{O}_{3}$, procurando mantener una dosis constante del mismo durante todo el tiempo de ozonización en cada evento de riego (entre 600 y $650 \mathrm{mV}$ a pH 6 en valor redox a la salida de la instalación). Con el fin de minimizar el efecto sobre la fertilización aplicada, la ozonización se dejó de aplicar en el periodo de abonado y post-abonado de cada riego, que se produjo siempre durante 50 minutos y 15 minutos respectivamente, dejando aproximadamente entre 120 y 135 minutos de riego con tratamiento de $\mathrm{O}_{3}$, dependiendo de la demanda evaporativa.

Para evaluar el comportamiento de la instalación de ozonización, se monitorizaron en continuo el potencial redox a la entrada y salida de la misma (sonda redox SEKO Mod SRH1). Para evaluar el comportamiento agronómico y fisiológico del cultivo, se realizaron durante el periodo de estudio, medidas periódicas de crecimiento de tronco y fruto, a partir de las cuales se obtuvieron las correspondientes tasas de crecimiento relativo y absoluto (RGR tronco y $A G R_{\text {fruto }}$ (Tattini 1995). También se determinaron los incrementos de peso fresco, seco y contenido de agua del fruto, así como el estado hídrico del cultivo (potencial hídrico de tallo a mediodía, $\Psi_{\text {tallo, }}$ mediante cámara de presión, Schölander et al., 1965) y el estado nutricional del cultivo, a partir de un muestreo realizado en el mes de noviembre. Los análisis de $\mathrm{Na}, \mathrm{K}$, 
$\mathrm{Cu}$ y $\mathrm{B}$ se han realizado mediante ICP (Varian MPX Vista Radial), el $\mathrm{Cl}^{-}$mediante cromatografía iónica (Dionex ICS 3000), el $\mathrm{N}$ total mediante un analizador elemental LECO FP-528. Los parámetros de producción (kilogramos por árbol, número de frutos y peso medio de fruto) y calidad al final (\% de zumo, pulpa y corteza, solidos solubles totales, acidez total, e índices de madurez, forma y color externo) de la campaña, que tuvo lugar a primeros de enero, también fueron evaluados, tomando en el momento de la cosecha, nueve frutos por árbol muestreado (en total 6 repeticiones por combinación Tipo de agua x Ozonización). El análisis estadístico de los datos se realizó mediante el análisis de la varianza (ANOVA) con el paquete STATGRAPHICS 5.1 plus y la separación de medias se realizó mediante el test de rango múltiple de Duncan.

Tabla 1. Análisis de las aguas de diferente naturaleza (Regenerada y Trasvase) utilizadas durante el periodo de estudio.

\begin{tabular}{|c|c|c|}
\hline \multirow{2}{*}{ Parámetro } & \multicolumn{2}{|c|}{ Tipo de agua } \\
\hline & Trasvase & Regenerada \\
\hline C.E (dS/m) & 1,16 & 3,16 \\
\hline $\mathrm{pH}$ & 8,14 & 7,92 \\
\hline $\mathrm{SS}(\mathrm{mg} / \mathrm{L})$ & 9,38 & 0,92 \\
\hline Turbidez (NTU) & 1,02 & 0,32 \\
\hline E. coli (UFC/100 mL) & 18 & 38 \\
\hline $\mathrm{Al}(\mathrm{mg} / \mathrm{L})$ & 0,11 & 0,05 \\
\hline$B(m g / L)$ & 0,04 & 0,65 \\
\hline $\mathrm{Ca}(\mathrm{mg} / \mathrm{L})$ & 79,32 & 139,1 \\
\hline $\mathrm{Fe}(\mathrm{mg} / \mathrm{L})$ & 0,05 & 0,06 \\
\hline $\mathrm{K}(\mathrm{mg} / \mathrm{L})$ & 3,51 & 36,3 \\
\hline Li (mg/L) & 0,01 & 0,11 \\
\hline $\mathrm{Mg}(\mathrm{mg} / \mathrm{L})$ & 29,08 & 76,53 \\
\hline $\mathrm{Mn}(\mathrm{mg} / \mathrm{L})$ & 0,21 & 0,49 \\
\hline $\mathrm{Na}(\mathrm{mg} / \mathrm{L})$ & 36,46 & 471,7 \\
\hline $\mathrm{Ni}(\mathrm{mg} / \mathrm{L})$ & $<0,01$ & 0,01 \\
\hline$P(m g / L)$ & $<0,1$ & 3,36 \\
\hline $\mathrm{Rb}(\mathrm{mg} / \mathrm{L})$ & $<0,01$ & 0,02 \\
\hline $\mathrm{Si}(\mathrm{mg} / \mathrm{L})$ & 2,62 & 4,89 \\
\hline$S(m g / L)$ & 73,11 & 220,8 \\
\hline $\mathrm{Sr}(\mathrm{mg} / \mathrm{L})$ & 1,33 & 2,56 \\
\hline $\mathrm{TI}(\mathrm{mg} / \mathrm{L})$ & $<0,01$ & 0,01 \\
\hline $\begin{array}{l}\text { As, Be, Bi, Cd, Co, Cr, } \\
\mathrm{Cu}, \mathrm{La}, \mathrm{Mo}, \mathrm{Pb}, \mathrm{Sb}, \mathrm{Se}, \\
\mathrm{Ti}, \mathrm{V}, \mathrm{Zn}(\mathrm{mg} / \mathrm{L})\end{array}$ & $<0,01$ & $<0,01$ \\
\hline
\end{tabular}

\section{4) Resultados y Discusión}

Durante el periodo de estudio (junio-agosto), se aplicaron para cada tratamiento: $134,44 \mathrm{~mm}$ y $134,94 \mathrm{~mm}$ de agua en Trasvase $(\mathrm{T})$ y Trasvase con ozono $\left(\mathrm{TO}_{3}\right)$ respectivamente y $138,01 \mathrm{~mm}$ y $111,51 \mathrm{~mm}$ en los tratamientos con agua regenerada sin (R) y con ozono $\left(\mathrm{RO}_{3}\right)$ respectivamente, con un total de 137 horas de generación de $\mathrm{O}_{3}$, en los tratamientos ozonizados. 
La diferente naturaleza de las aguas utilizadas, destacando valores significativamente más elevados de $E$. coli, $\mathrm{Na}, \mathrm{B}, \mathrm{S}, \mathrm{Ca}$ y $\mathrm{Mg}$ en las aguas regeneradas respecto a las del Trasvase (Tabla 1), se reflejó en los valores redox de las mismas, durante los eventos de riego en los tratamientos $\sin \mathrm{O}_{3}$, para cada tipo de agua $(285 \mathrm{mV}$ y $360 \mathrm{mV}$, en agua regenerada y agua del Trasvase respectivamente (Tabla 2).

Los resultados del funcionamiento de la instalación de ozonización, a partir de la evaluación de los niveles redox alcanzados durante los eventos de riego, mostraron comportamientos muy diferentes, según el tipo de agua empleada. El sistema, fue capaz de producir la ozonización para ambos tipos de aguas, alcanzando niveles de potencial redox significativamente superiores a la salida de la instalación, después de la ozonización, para ambos tipos de agua (Tabla 2). Sin embargo, mientras con agua del Trasvase el sistema fue capaz de mantener el nivel de potencial redox a la salida dentro de los umbrales marcados en el sistema (589 mV), cuando se utilizó agua regenerada, el sistema no llegó a alcanzarlos (477 mV) (Tabla 2 y Figura 2), lo que pudo ser debido a la carga orgánica presente en este tipo de aguas, entre otros parámetros, muy diferente al agua del Trasvase (Tabla 1).

Tabla 2. Valores medios de potencial redox a la entrada $\left(\sin \mathrm{O}_{3}\right)$ y salida $\left(\operatorname{con} \mathrm{O}_{3}\right)$ de la instalación durante el periodo de aplicación de $\mathrm{O}_{3}$ en los eventos de riego durante el periodo de estudio con aguas de diferente naturaleza.

\begin{tabular}{cccc}
\hline \multicolumn{4}{c}{ Potencial redox $(\mathbf{m V})$} \\
Tipo de agua & Entrada & Salida & ANOVA \\
\hline Regenerada & 285 & 477 & $* * *$ \\
Trasvase & 360 & 589 & $* * *$ \\
ANOVA & $* *$ & $* * *$ & \\
\hline${ }^{* * *}$ y $n$ indican diferencias significativas a $P<0,01 ; P<0,001$ & y & no significativas \\
spectivamente, según el test de Rango Múltiple de Duncan. &
\end{tabular}
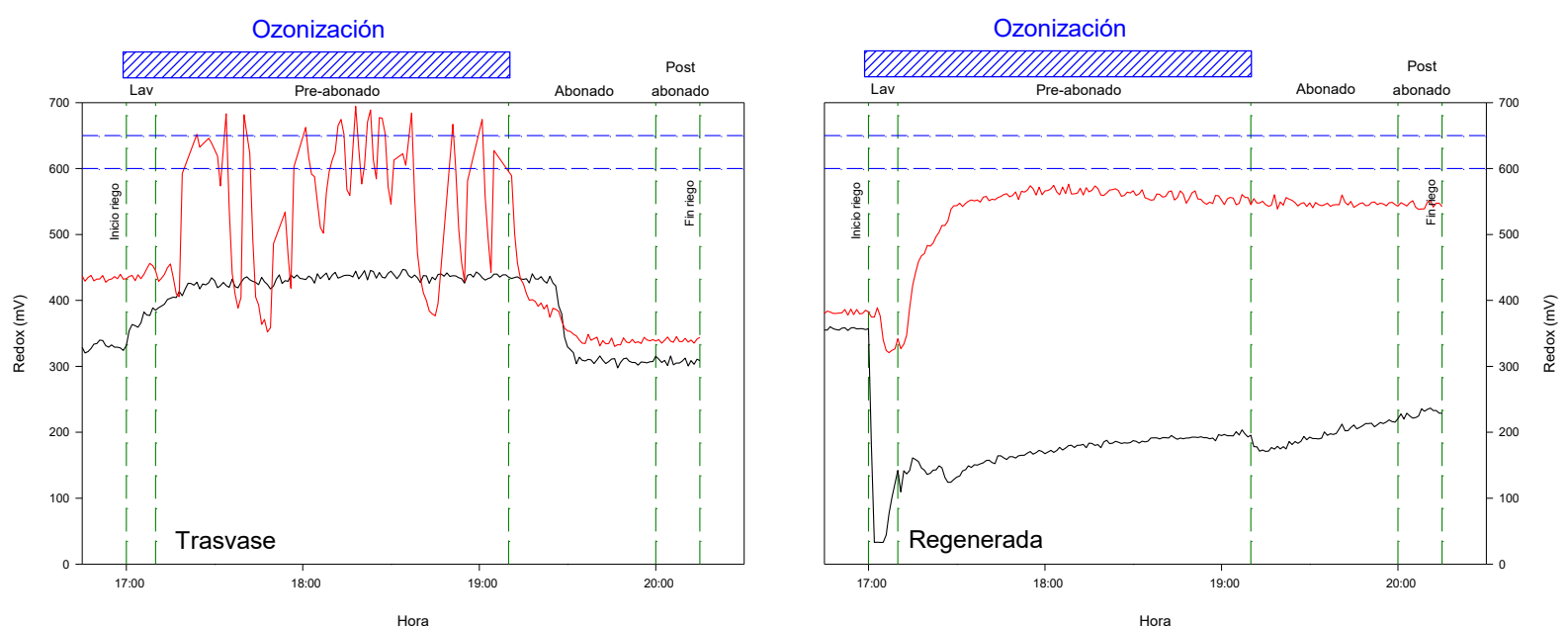

Figura 2. Evolución de los valores de potencial redox durante los eventos de riego en tratamientos de ozonización con agua de Trasvase y regenerada, en días representativos para cada tipo de agua. Color de líneas: rojo, redox salida; negro, redox entrada; azul umbrales sistema redox; verde se delimitan los eventos durante el riego. 
Además, durante los periodos de ozonización con agua regenerada, la instalación mantuvo un régimen de presiones inferior que con agua del Trasvase, afectando de manera significativa al caudal de descarga de los goteros. Esta situación, produjo una reducción del agua aplicada en este tratamiento $\left(\mathrm{RO}_{3}\right)$ de un $17 \%$ inferior al resto de tratamientos. Esto explicaría, los menores valores alcanzados de potencial hídrico de tallo a mediodía en este tratamiento al final del periodo, $-1,94 \mathrm{MPa}$, que fueron significativamente inferiores al alcanzado por el tratamiento de agua regenerada sin $\mathrm{O}_{3},-1,65 \mathrm{MPa}$ (Tabla 3).

Tabla 3. Parámetros de crecimiento vegetativo y del fruto, y estado hídrico del cultivo durante el periodo de estudio (entre los meses de julio y agosto). Estado hídrico al final del periodo.

\begin{tabular}{|c|c|c|c|c|c|}
\hline \multirow{3}{*}{ Factores } & \multicolumn{4}{|c|}{ PARÁMETROS CRECIMIENTO } & \multirow{3}{*}{ 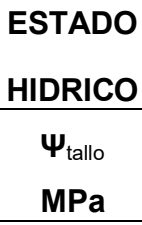 } \\
\hline & \multirow{2}{*}{$\begin{array}{c}\text { Tronco } \\
\text { RGR } \\
\text { cm cm }_{\text {Tronco }}^{-1} \text { dia }^{-1}\end{array}$} & \multicolumn{3}{|c|}{ Fruto } & \\
\hline & & $\begin{array}{c}\text { AGR } \text { Diámetro } \text { mm día-1 }^{-1} \\
\end{array}$ & $\begin{array}{c}\text { AGR Peso fresco } \\
\text { g día }{ }^{-1}\end{array}$ & $\begin{array}{c}\text { Agua } \\
\text { acumulada } \\
\text { g día-1 }^{-1}\end{array}$ & \\
\hline \multicolumn{6}{|l|}{ Tipo de Agua } \\
\hline Trasvase & 1,35E-05 & 0,297 & $1,46 b$ & $1,24 b$ & $-1,84$ \\
\hline Regenerada & 1,46E-05 & 0,280 & $1,22 \mathrm{a}$ & $1,04 \mathrm{a}$ & $-1,79$ \\
\hline \multicolumn{6}{|l|}{ Ozonización } \\
\hline Ozono SI & $1,18 \mathrm{E}-05$ & 0,279 & 1,41 & $1,20 b$ & $-1,86$ \\
\hline Ozono NO & 1,63E-05 & 0,298 & 1,27 & $1,08 \mathrm{a}$ & $-1,77$ \\
\hline \multicolumn{6}{|l|}{ Tipo agua x Ozonización } \\
\hline $\mathrm{T}$ & $2,15 \mathrm{E}-05 \mathrm{~b}$ & 0,283 & $1,52 b$ & $1,29 c$ & $-1,88$ a \\
\hline $\mathrm{TO}_{3}$ & 5,45E-06 a & 0,312 & $1,40 \mathrm{~b}$ & $1,19 b$ & $-1,79 a b$ \\
\hline $\mathrm{R}$ & $1,11 \mathrm{E}-05 \mathrm{ab}$ & 0,313 & $1,02 \mathrm{a}$ & $0,87 \mathrm{a}$ & $-1,65 b$ \\
\hline $\mathrm{RO}_{3}$ & $1,82 \mathrm{E}-05 \mathrm{ab}$ & 0,247 & $1,42 b$ & $1,22 \mathrm{bc}$ & $-1,94 a$ \\
\hline \multicolumn{6}{|l|}{ ANOVA } \\
\hline Tipo de Agua & ns & ns & * & * & ns \\
\hline Ozonización & ns & ns & ns & * & ns \\
\hline Tipo agua x Ozonización & * & ns & * & * & $* *$ \\
\hline
\end{tabular}

Por otra parte, y aunque el periodo en el que se aplicó el $\mathrm{O}_{3}$ en este estudio para este cultivo se caracteriza por una parada del crecimiento vegetativo en beneficio del crecimiento del fruto, se observó, entre julio y agosto, una disminución significativa de la tasa de crecimiento del perímetro del tronco ( $R G R_{\text {Tronco }}$ ), en el tratamiento con agua de buena calidad ozonizada $\left(\mathrm{TO}_{3}\right)$ respecto a este mismo tipo de aguas no ozonizadas ( $\mathrm{T}$ ) (Tabla 3), lo que no fue observado para aguas regeneradas $(R)$. En este mismo periodo, el crecimiento del diámetro del fruto $\left(A G R_{\text {Fruto }}\right)$, no experimentó diferencias entre tratamientos, sin embargo, si se apreció una disminución significativa del incremento del peso fresco (AGR Peso fresco) $_{\text {) }}$ los frutos del tratamiento de agua regenerada sin ozonizar $(R)$, respecto al resto de tratamientos, que estuvo asociado a una menor acumulación de agua en el fruto en este tratamiento (Tabla 3 ). En general, las aguas regeneradas durante este periodo, produjeron una disminución del peso fresco del fruto, asociada a una menor cantidad de agua acumulada en el tratamiento no ozonizado, por lo que la ozonización en este caso parece aportar una ventaja respecto a la acumulación de agua en el fruto en estas condiciones. 
Un estado hídrico más favorable durante el periodo de estudio, en el tratamiento $R$ que el resto de tratamientos, pudo favorecer que el crecimiento vegetativo no se viera afectado en esta etapa, en detrimento de la acumulación de agua en el fruto.

La aplicación del tratamiento de ozonización, para ambos tipos de aguas, durante uno de los periodos más sensibles en el cultivo de pomelo (Fase II de crecimiento del fruto), produjo una ligera disminución de los parámetros productivos, si bien dicha reducción, (de apenas un $7 \%$ ), solo fue significativa para el peso total por árbol (Tabla 4). No se apreciaron diferencias significativas respecto al Tipo de agua, resultados similares a los reportados en estas condiciones en este mismo cultivo por Pedrero et al. (2015), aunque otros autores, si han destacado después de cinco años de riego con aguas regeneradas en pomelo, una disminución en los parámetros fisiológicos y en la producción (Romero-Trigueros et al., 2014). El tratamiento $\mathrm{RO}_{3}$, que presentó los mayores niveles de estrés durante esta fase $(-1,94 \mathrm{MPa})$, también fue el que obtuvo los valores más bajos de producción, aunque no de manera estadísticamente significativa. Con aguas de buena calidad, en pomelo 'Star Ruby', una reducción de la cosecha, cuando se aplicó un estrés hídrico moderado, durante esta fase de crecimiento de fruto, ha sido reportado por Perez-Pérez et al. (2014).

Tabla 4. Parámetros productivos durante la campaña 2019-2020

\begin{tabular}{rccc} 
Factor & $\begin{array}{c}\mathbf{N}^{\circ} \\
\text { Frutos }\end{array}$ & $\begin{array}{c}\text { Peso } \\
\text { (kg/árbol) }\end{array}$ & $\begin{array}{c}\text { P medio } \\
\text { fruto }(\mathbf{g})\end{array}$ \\
\hline Tipo de Agua & & & \\
Trasvase & 810 & 249,0 & 307,1 \\
Regenerada & 796 & 247,1 & 312,0 \\
Ozonización & & & \\
Ozono SI & 793 & 239,3 a & 303,7 \\
Ozono NO & 814 & $256,7 \mathrm{~b}$ & 315,4
\end{tabular}

Tipo agua x Ozonización

$\begin{array}{rlll}\mathrm{T} & 827 & 253,4 & 306,5 \\ \mathrm{TO}_{3} & 794 & 244,6 & 307,7 \\ \mathrm{R} & 801 & 260,1 & 324,3 \\ \mathrm{RO}_{3} & 792 & 234,0 & 299,7\end{array}$

\begin{tabular}{|c|c|c|c|}
\hline \multicolumn{4}{|l|}{ ANOVA } \\
\hline Tipo de Agua & ns & ns & ns \\
\hline Ozonización & ns & * & ns \\
\hline Tipo agua x Ozonización & ns & ns & ns \\
\hline
\end{tabular}

Por otra parte, el Tipo de agua afectó principalmente a la calidad de los frutos y a ciertos aspectos nutricionales del cultivo. Así, las aguas regeneradas, produjeron frutos de calidad inferior (menos \% de zumo, y más \% de pulpa y corteza, aunque estos dos últimos parámetros no de manera estadísticamente significativa) (Tabla 5). En este sentido, RomeroTrigueros et al. (2017), en condiciones muy similares, han reportado resultados diferentes, no encontrando diferencias en el porcentaje de zumo entre ambos tipos de agua. Con aguas de buena calidad, un estrés hídrico moderado, en esta fase y para este mismo cultivo, produjo también una disminución de la calidad de los frutos, con valores de $\%$ de zumo e índice de color externo inferiores al control (Pérez-Pérez et. al., 2014), como los observados en nuestros resultados con aguas regeneradas.

Por otra parte, las aguas regeneradas presentaron valores ligeramente superiores de boro, por encima de 100 ppm, umbral considerado tóxico para este cultivo (Legaz et al., 1995), y ligeramente inferiores a los reportados por otros autores utilizando aguas regeneradas en cítricos (Maurer et al., 1995; Reboll et al., 2000), y valores también superiores de cloruro en hoja (Tabla 6), aunque muy inferiores al $1 \%$ considerado como tóxico para cultivos sensibles (Ayers y Westcot, 1985). Por otra parte, la ozonización, produjo frutos ligeramente más 
achatados (valores de Índice de forma inferiores, Tabla 5). Además se encontraron diversas interacciones significativas en algunos de estos parámetros. Así, la ozonización de aguas regeneradas produjo frutos significativamente menos coloreados que el resto de tratamientos (Tabla 4), y disminuciones significativas respecto al tratamiento no ozonizado de $\mathrm{K}, \mathrm{N}$ total y $\mathrm{Na}$ en hoja, y aunque no de manera significativa también de $\mathrm{Cu}$.

Tabla 5. Parámetros de calidad del fruto de la cosecha 2019-2020.

\begin{tabular}{|c|c|c|c|c|c|c|c|c|}
\hline Factor & $\%$ Zumo & $\begin{array}{c}\% \\
\text { Pulpa }\end{array}$ & $\begin{array}{c}\% \\
\text { corteza }\end{array}$ & $\begin{array}{c}\text { SST } \\
\text { ( }{ }^{\circ} \text { Brix) }\end{array}$ & $\begin{array}{c}\text { Acidez } \\
\text { (g/L) }\end{array}$ & $\begin{array}{c}\text { Índice } \\
\text { Madurez }\end{array}$ & $\begin{array}{l}\text { Índice } \\
\text { forma }\end{array}$ & $\begin{array}{l}\text { Índice } \\
\text { color } \\
\text { externo }\end{array}$ \\
\hline \multicolumn{9}{|l|}{ Tipo de Agua } \\
\hline Trasvase & $48,6 \mathrm{~b}$ & 3,2 & 48,0 & 9,9 & 25,4 & 3,9 & 0,87 & $6,5 \mathrm{~b}$ \\
\hline Regenerada & $47,2 \mathrm{a}$ & 3,4 & 49,2 & 10,1 & 25,4 & 4,0 & 0,87 & $5,6 \mathrm{a}$ \\
\hline \multicolumn{9}{|l|}{ Ozonización } \\
\hline Ozono SI & 48,4 & 3,4 & 48,1 & 10,1 & 25,6 & 3,9 & $0,86 a$ & 5,9 \\
\hline Ozono NO & 47,4 & 3,2 & 49,2 & 9,9 & 25,1 & 3,9 & $0,88 \mathrm{~b}$ & 6,2 \\
\hline \multicolumn{9}{|l|}{ Tipo agua x Ozonización } \\
\hline $\mathrm{T}$ & 48,1 & 3,2 & 48,5 & 9,9 & 25,0 & 4,0 & 0,88 & $6,2 \mathrm{~b}$ \\
\hline $\mathrm{TO}_{3}$ & 49,1 & 3,2 & 47,5 & 10,0 & 25,9 & 3,9 & 0,86 & $6,8 b$ \\
\hline $\mathrm{R}$ & 46,8 & 3,2 & 49,8 & 10,0 & 25,3 & 3,9 & 0,88 & $6,3 \mathrm{~b}$ \\
\hline $\mathrm{RO}_{3}$ & 47,6 & 3,5 & 48,6 & 10,2 & 25,4 & 4,0 & 0,86 & $5,0 \mathrm{a}$ \\
\hline \multicolumn{9}{|l|}{ ANOVA } \\
\hline Tipo de Agua & * & ns & ns & ns & ns & ns & ns & * \\
\hline Ozonización & ns & ns & ns & ns & ns & ns & * & ns \\
\hline Tipo agua x Ozonización & ns & ns & ns & ns & ns & ns & ns & * \\
\hline
\end{tabular}

* y $n$ s indican diferencias significativas a $P<0,05$ y no significativas respectivamente, según el test de Rango Múltiple de Duncan. Para cada columna, valores con la misma letra, no son significativamente diferentes al $95 \%$.

Tabla 6. Resultados estado nutricional del cultivo. Campaña 2019-210

\begin{tabular}{|c|c|c|c|c|c|c|}
\hline Factor & B (ppm) & Cu (ppm) & K (\%) & $\mathrm{Na}(\%)$ & $\mathrm{Cl}(\%)$ & $\mathbf{N}(\%)$ \\
\hline \multicolumn{7}{|l|}{ Tipo de Agua } \\
\hline Trasvase & $93,1 \mathrm{a}$ & 16,7 & 0,68 & 0,08 & $0,12 \mathrm{a}$ & 2,46 \\
\hline Regenerada & $103,0 \mathrm{~b}$ & 15,4 & 0,67 & 0,08 & $0,13 b$ & 2,49 \\
\hline \multicolumn{7}{|l|}{ Ozonización } \\
\hline Ozono SI & 95,5 & 16,3 & 0,66 & 0,08 & 0,13 & 2,47 \\
\hline Ozono NO & 100,6 & 15,7 & 0,69 & 0,08 & 0,13 & 2,49 \\
\hline \multicolumn{7}{|l|}{ Tipo agua x Ozonización } \\
\hline$T$ & 95,2 & $14,67 \mathrm{a}$ & $0,66 a b$ & $0,07 \mathrm{a}$ & 0,12 & $2,44 \mathrm{a}$ \\
\hline $\mathrm{TO}_{3}$ & 90,9 & $18,69 \mathrm{~b}$ & $0,71 \mathrm{~b}$ & $0,09 a b$ & 0,12 & $2,48 a b$ \\
\hline $\mathrm{R}$ & 106,1 & $16,73 a b$ & $0,72 \mathrm{~b}$ & $0,10 \mathrm{~b}$ & 0,14 & $2,53 \mathrm{~b}$ \\
\hline $\mathrm{RO}_{3}$ & 99,9 & $13,97 \mathrm{a}$ & $0,61 \mathrm{a}$ & $0,07 \mathrm{a}$ & 0,13 & $2,45 a$ \\
\hline \multicolumn{7}{|l|}{ ANOVA } \\
\hline Tipo de Agua & * & ns & ns & ns & * & ns \\
\hline Ozonización & ns & ns & ns & ns & ns & ns \\
\hline Tipo agua x Ozonización & ns & $* *$ & * & ** & ns & * \\
\hline
\end{tabular}

\section{5) Conclusiones y Recomendaciones}

Nuestros resultados denotan que las instalaciones utilizadas para la aplicación de un tratamiento de ozonización a pie de parcela, cuando se utilizan aguas regeneradas, pueden presentar dificultades derivadas de la naturaleza de estas aguas (elevados niveles de materia orgánica y otras sustancias altamente oxidables), lo que hace necesario un estudio más exhaustivo de estos sistemas para poder llevar a cabo un tratamiento eficaz de $\mathrm{O}_{3}$ en estas 
condiciones. Dimensionar adecuadamente la generación de $\mathrm{O}_{3}$ para cada instalación en función de la naturaleza de las aguas utilizadas e implementar sistemas que garanticen la presurización de la instalación y procuren la dosificación adecuada de medio ácido, pueden ser algunas de las recomendaciones a seguir para este tipo de instalaciones.

Por otra parte, la respuesta agronómica y fisiológica, ante estos tratamientos requiere igualmente de un mayor y más largo estudio, para poder alcanzar resultados confiables, dado que en el presente estudio solo se evaluaron los efectos de la aplicación de estos tratamientos durante una etapa muy concreta del desarrollo del fruto.

\section{6) Agradecimientos}

El trabajo ha sido financiado por el proyecto RIS3MUR REUSAGUA, financiado por la Consejería de Empresa, Industria y Portavocía de la Región de Murcia, en el marco del Programa Operativo FEDER 2014-2020.

\section{7) Bibliografía}

Abuarab, M., Mostafa, E., \& Ibrahim, M. (2013). Effect of air injection under subsurface drip irrigation on yield and water use efficiency of corn in a sandy clay loam soil. Journal of Advanced Research, 4(6), 493-499.

Assouline, S., Narkis, K., 2013. Effects of long-term irrigation with treated wastewater on the root zone environment. Vadose Zone J

Ayers, R. S. y Westcot, D.W., 1985. Water quality for agriculture. FAO Irrigation and Drainage Paper 29. Rome (Reprinted, 1989, 1994).

Bhattarai, S. P., and Midmore, D. J. (2004). Oxygation of rhizosphere with subsurface aerated irrigation water improves lint yield and performance of cotton on saline heavy clay soil. In "4th International Crop Science Congress," Brisbane, Australia.

Bhattarai, S., Pendergast, L., and Midmore, D. J. (2005). Oxygation of subsurface drip irrigated tomato (Lycopersicon esculentum L.) improves yield performance, tolerance to salinity and water use efficiency in normal and saline heavy clay soil. Sci. Hortic.

Gerrity, D., \& Snyder, S. (2011). Review of Ozone for Water Reuse Applications: Toxicity, Regulations, and Trace Organic Contaminant Oxidation. Ozone: Science \& Engineering, 33(4), 253-266.

Kim, I. H., Yamashita, N., Kato, Y., \& Tanaka, H. (2009). Discussion on the application of $\mathrm{UV} / \mathrm{H}(2) \mathrm{O}(2), \mathrm{O}(3)$ and $\mathrm{O}(3) / \mathrm{UV}$ processes as technologies for sewage reuse considering the removal of pharmaceuticals and personal care products. Water Science and Technology, 59(5), 945-55.

Letey, J. 1961. Aeration, compaction and drainage. Calif. Turf Grass Culture 11,17-21.

Legaz, F., Serna, M.D., Ferrer, P., Cebolla V. and Primo-Millo, E., 1995. Análisis de hojas, suelos y águas para el diagnóstico nutricional de plantaciones de cítricos. Procedimiento de toma de muestras. Generalitat Valenciana.

Martínez, S., Suay, R., Moreno, J., \& Segura, M. L. (2013). Reuse of tertiary municipal wastewater effluent for irrigation of Cucumis melo L. Irrigation Science, 31(4), 661-672. http://doi.org/10.1007/s00271-012-0342-4 
Maurer, M.A., Davies, F.S., and Graetz, D. A., 1995. Reclaimed wastewater irrigation of reser Marsh grapefruit trees. Hortucultiral Sciencie Dept., Soil and Water Science Dept. University of Florida, Gainesville, Fla. 32611. Proc.Fla. State Hort. Soc. 108:93-99

Pérez-Pérez, J.G.; Robles, J.M.; Botía, P. 2014. Effects of deficit irrigation in different fruit growth stages on 'Star Ruby' grapefruit trees in semi-arid conditions. Agricultural Water Management. 133:44-54.

Poysa VW, Tan CS, Stone JA. Flooding stress and the root development of several tomato genotypes. Hort Sci 1987;22:24-6.

Reboll, V., Cerezo, M., Roig, A., Flors,V., Lapeña, V. and García-Agustín, P., 2000. Influene of wastewater vs groundwater on young Citrus trees. J. Sci. Food Agric. 80: 1441-1446.

Resh, 1997. Cultivos Hidropónicos, 4th ed., 509 pp., Ediciones MundiPrensa, Madrid, Spain

Romero-Trigueros, C., Nortes Tortosa, P.A., Pedrero Salcedo, F., Mounzer, O., Alarcón Cabañero, J.J., Bayona Gambín, J.M., Nicolás Nicolás, E., 2014a. Assessment of the viability of using saline reclaimed water in grapefruit in medium to long term. Spanish Journal of Agricultural Research, 12(4): 1137-1148. doi: 10.5424/sjar/2014124-5495

Romero-Trigueros, C. \& Parra, Margarita \& Bayona, Jose \& Nortes, Pedro \& Alarcón, J.J. \& Nicolás, E.. (2017). Effect of deficit irrigation and reclaimed water on yield and quality of grapefruits at harvest and postharvest. LWT - Food Science and Technology. 405-4011. 10.1016/j.Iwt.2017.05.001.

Seguí, L.A. 2004. Sistemas de regeneración y reutilización de aguas residuales. Metodología para el análisis Técnico-Económico y casos. Tesis Doctoral, Universidad Politécnica de Cataluña, España. 256pp.

Segura ML, Granados MR, Jiménez S (2003) Reutilización de agua residual para riego de judía verde: influencia sobre la producción y concentración de nutrientes en hoja. X Congreso Nacional de Ciencias Hortícolas, Pontevedra, Spain. Actas de Horticultura 39:621-623

Schölander, P., Hammel, H., Bradstreet, E. and Hemmingsen, E. (1965). Sap pressure in vascular plants. Science 148, 339-345.

Tattini M, Gucci R, Romani A, Baldi A, Everard JD (1995) Growth, gas exchange and ion content in Olea europaea plants during salinity and subsequent relief. Physiol Plant 95:203-210. 